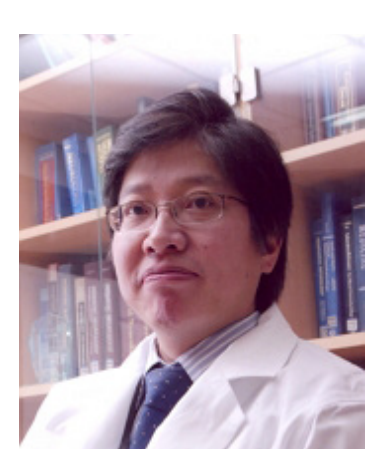

\section{Congratulatory remarks on expanding the research field for encephalitis and neuroinflammation}

\author{
Sang Kun Lee \\ Department of Neurology, Laboratory for Neurotherapeutics, Comprehensive Epilepsy Center, Seoul \\ National University Hospital, Seoul, Korea
}

On behalf of all members of our society, it is my great pleasure and privilege to congratulate the publication of the first issue of encephalitis. The field of encephalitis and neuroinflammation is an extraordinary field of neuroscience that gives us a sense of awe. It includes various kinds of encephalitis and altered neuroimmunology diseases with remarkable clinical characteristics, which gives us very important insights into neuroscience regardless of the basic and clinical aspects.

Recently, many new categories of encephalitis have been found and the efforts to discover novel forms of this disease are also ongoing. With these discoveries and their associated efforts, we can begin to grasp the secrets of neuroimmunology and the functions and the connections between various neural systems macroscopically and microscopically. We, therefore, are able to refine the diagnosis and treatment of various encephalitis and neuroinflammation diseases and step forward to save the lives of patients and nervous system function.

Many more studies should provide us with a greater under- standing of the mechanisms of these diseases and aid in further treatment strategy developments at the state of the art level. Korea is one of the leading countries doing cutting edge research to develop diagnosis and treatment guidelines for encephalitis diseases. As a result, we need a more suitable vehicle such as $\boldsymbol{e n}$ cephalitis to reach these various goals. encephalitis is dedicated to original research that encompasses basic and clinical fields that investigate encephalitis, neuroinflammation, and neuroimmunology. Case reports are also welcome. All the members of the Editorial Board and publication staff are looking forward to receiving a wide range of submissions and contributions from all international researchers and clinicians. Thank you very much.

Warm regards,

Sang Kun Lee

Honorary President,

Korean Encephalitis and Neuroinflammation Society

Received: December 28, 2020 Accepted: December 31, 2020

Correspondence: Sang Kun Lee

Department of Neurology, Laboratory for Neurotherapeutics, Comprehensive Epilepsy Center, Seoul National University Hospital, 101 Daehak-ro, Jongno-gu, Seoul 03080, Korea

E-mail: sangkun2923@gmail.com

ORCID: https://orcid.org/0000-0003-1908-0699

Copyright (C) 2021 by The Korean Encephalitis and Neuroinflammation Society

This is an open access article distributed under the terms of the Creative Commons Attribution Non-Commercial License (http://creativecommons.org/licenses/by-nc/4.0/) which permits unrestricted non-commercial use, distribution, and reproduction in any medium, provided the original work is properly cited. 